\title{
Spectrally Efficient Alamouti Code Structure in Asynchronous Cooperative Systems
}

\author{
Mojtaba Rahmati and Tolga M. Duman, Fellow, IEEE
}

\begin{abstract}
A relay communication system with two amplify and forward (AF) relays under flat fading channel conditions is considered where the signals received from the relays are not necessarily time aligned. We propose a new time-reversal (TR)-based scheme providing an Alamouti code structure which needs a smaller overhead in transmitting every pair of data blocks in comparison with the existing schemes and, as a result, increases the transmission rate significantly (as much as $\mathbf{2 0 \%}$ ) in exchange for a small performance loss. The scheme is particularly useful when the delay between the two relay signals is large, e.g., in typical underwater acoustic (UWA) channels.
\end{abstract}

Index Terms-Alamouti scheme, asynchronous communications, cooperative systems.

\section{INTRODUCTION}

A S IT is well known the Alamouti scheme [1] is a two antenna transmit diversity scheme for multi-input multioutput (MIMO) communication systems. Although it was originally proposed for centralized MIMO systems, the idea was later extended to cooperative communications [2]. In most existing related literature, the actively cooperating nodes are assumed to be perfectly time aligned. Due to the distributed nature of a cooperative system, achieving perfect time alignment among the signals received from geographically separated nodes may not always be feasible. Therefore, conventional MIMO transmission schemes designed for synchronized transmitting antennas may not be directly applicable.

There are existing results in the literature to achieve diversity in asynchronous cooperative systems with decode and forward (DF) relaying, e.g., [3]-[5]. In [3], time reversal spacetime block coding (TR-STBC) and in [5] distributed linear convolutional space-time codes (DLC-STC) are proposed where each transmitted block is preceded by a time guard greater than the maximum possible relative delay $D_{\max } T$ among the relays assuming that the relative delays are integer multiples of the sampling time $T$. There are also a few works on AF relaying, e.g., [6]-[8]. In [6], an Alamouti scheme employing orthogonal frequency division multiplexing (OFDM) is proposed in

Manuscript received November 25, 2013; revised January 31, 2014; accepted February 09, 2014. Date of publication February 25, 2014; date of current version March 10, 2014. This work was supported by Qatar National Research Fund (QNRF) grant through National Priority Research Program (NPRP) under Grant 09-242-2-099. QNRF is an initiative of Qatar Foundation. The associate editor coordinating the review of this manuscript and approving it for publication was Prof. Ron Dabora.

M. Rahmati is with the School of Electrical, Computer and Energy Engineering (ECEE) of Arizona State University, Tempe, AZ 85287-5706 USA (e-mail: mojtaba@asu.edu).

T. M. Duman is with the Department of Electrical and Electronics Engineering, Bilkent University, Bilkent, Ankara 06800, Turkey, on leave from the School of Electrical, Computer and Energy Engineering (ECEE) of Arizona State University, Tempe, AZ 85287-5706 USA (e-mail: duman@ee.bilkent.edu.tr).

Color versions of one or more of the figures in this paper are available online at http://ieeexplore.ieee.org.

Digital Object Identifier 10.1109/LSP.2014.2308296 which the transmitted OFDM blocks are separated by adding a cyclic prefix (CP) longer than $D_{\max } T$ and at the AF relays, time reversal and complex conjugation operations are implemented. However, as $D_{\max }$ becomes large (as in a typical UWA channel), the effective transmission rate is diminished. That is, in transmitting binary phase shift keying (BPSK) modulated data blocks of length $M$, the maximum achievable spectral efficiency is $\frac{M}{M+D_{m a x}}$. In [8], the joint channel and timing error estimation is considered for AF asynchronous cooperative systems with relative delays less than one sampling time.

In this letter, we consider an asynchronous cooperative system with two AF relays where only the receiver is aware of the relative time delay between the relays. Our main objective is to reduce the overhead needed to alleviate the asynchronism issues in cooperative communications. To this end, we propose a new single carrier (SC)-TR-STBC scheme which needs a smaller overhead in comparison with the existing solutions to combat the asynchronism issues and, as a result, increases the effective transmission rate. We focus on the case of a single tap (frequency flat) channel, and utilize superposition of two suffixes generated for the first and second blocks in between the two transmitted blocks. This results in a more efficient way of obtaining the Alamouti structure at the receiver than the existing schemes (e.g., [3]), however, some symbols get corrupted by intersymbol interference (ISI). The good news is that the interfering symbols are the ones with the Alamouti coding structure without ISI, therefore we can first detect these symbols then use the symbol estimates to detect the rest of the symbols. To accomplish this goal, at the transmitter, both blocks are transmitted prefix-free and suffix-free, and they are separated by a time guard not less than $D_{\max } T$. The relay nodes produce the cyclic suffix for the first and $\mathrm{CP}$ for the second block and forward the superposition of the noisy $\mathrm{CP}$ and cyclic suffix to the destination while at the same time they perform appropriate time reversal and complex conjugation. The overhead is only $D_{\max }$ and as a result in transmitting BPSK modulated data blocks of length $M$ the maximum achievable spectral efficiency is $\frac{2 M}{2 M+D_{\max }}$, e.g., for $D_{\max }=\frac{M}{2}$, transmission rate is increased by $20 \%$ in comparison with the existing schemes.

The letter is organized as follows. In Section II, we present the considered asynchronous cooperative communication system model. In Section III, we introduce the proposed relaying scheme. In Section IV, we first obtain the optimal detector structure, and then present a simpler sub-optimal detector which results in a fast maximum likelihood (ML) detector for all the symbols. We provide numerical results in Section $\mathrm{V}$, and finally conclude the letter in Section VI.

\section{SYSTEM MODEL}

A cooperative relay system with two AF relays, shown in Fig. 1 is considered in which the channels from the source to the relays, i.e., $h_{i}$, and the relays to the destination, i.e., $g_{i}$, are all independent quasi-static Rayleigh fading (constant over two transmitted 


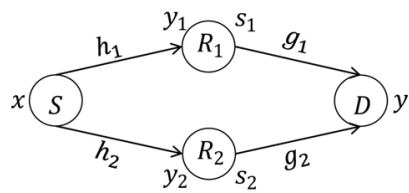

Fig. 1. Relay channel with two relays.

TABLE I

RELAYING StRATEGy OF THE RELAY NODES

\begin{tabular}{|c|c|c|c|}
\hline & $\begin{array}{c}\text { First block } \\
\boldsymbol{s}_{i, 1}\end{array}$ & $\begin{array}{c}\text { Infix block } \\
\boldsymbol{s}_{i, g}\end{array}$ & $\begin{array}{c}\text { Second block } \\
\boldsymbol{s}_{i, 2}\end{array}$ \\
\hline$R_{1}$ & $\boldsymbol{y}_{1,1}$ & $\boldsymbol{y}_{1,1}\left[0: M_{p}-1\right]+\boldsymbol{y}_{1,2}\left[M-M_{p}: M-1\right]$ & $\boldsymbol{y}_{1,2}$ \\
\hline$R_{2}$ & $\zeta\left(\boldsymbol{y}_{2,2}^{*}\right)$ & $-\zeta\left(\boldsymbol{y}_{2,1}^{*}\left[0: M_{p}-1\right]\right)+\zeta\left(\boldsymbol{y}_{2,2}^{*}\left[M-M_{p}: M\right]\right)$ & $-\zeta\left(\boldsymbol{y}_{2,1}^{*}\right)$ \\
\hline
\end{tabular}

data blocks), and there is no direct link between the source and destination. We assume that the source node transmits raised-cosine waveforms with symbol period $T$ and roll-off factor $\beta$. We further assume that $s_{2}$ is received $(D+d) T(|D| \in\{0,1, \cdots\}$ and $|d| \leq 0.5)$ seconds later than $s_{1}$ and assume that the maximum possible length of the delay is $D_{\max } T$, i.e., $|D+d| \leq D_{\max }$, where $D_{\max }$ is known at the transmitter side.

\section{Proposed Signaling Scheme}

\section{A. Source Node Signaling Approach}

Let us consider transmission of $2 B$ blocks of length $M$ over a channel with $2 D_{\max } \leq M$. At the transmitter, a SC transmission is performed for which two consecutive blocks, $\boldsymbol{x}_{2 b+1}$ and $\boldsymbol{x}_{2 b+2}(b=\{0, \cdots, B-1\})$, are separated by a time guard of length $M_{p} T\left(M_{p} \geq D_{\max }\right)$. Without loss of generality we focus on transmission of two blocks $\boldsymbol{x}_{1}=\left[x_{1,0}, \cdots, x_{1, M-1}\right]^{T}$ and $\boldsymbol{x}_{2}=\left[x_{2,0}, \cdots, x_{2, M-1}\right]^{T}$, therefore the objective is transmission of $\boldsymbol{x}=\left[\boldsymbol{x}_{1}^{T}, \boldsymbol{x}_{2}^{T}\right]^{T}$. However, the block $\overline{\boldsymbol{x}}=\left[\boldsymbol{x}_{1}^{T}, 0_{1 \times M_{p}}, \boldsymbol{x}_{2}^{T}\right]^{T}$ is transmitted over the channel, where $0_{n \times m}$ denotes the $n$ by $m$ all zero matrix. Note that instead of zero padding, one can also transmit the CP of the first block or cyclic suffix of the second block as a time guard, however, we focus on zero padding approach in this letter. The transmitted block $\overline{\boldsymbol{x}}$ can also be written as

$$
\overline{\boldsymbol{x}}=\boldsymbol{Q} \boldsymbol{x}=\left[\begin{array}{cc}
\boldsymbol{I}_{M} & \mathbf{0}_{M \times M} \\
\mathbf{0}_{M_{p} \times M} & \mathbf{0}_{M_{p} \times M} \\
\mathbf{0}_{M \times M} & \boldsymbol{I}_{M}
\end{array}\right] \boldsymbol{x},
$$

with $\boldsymbol{I}_{M}$ denoting the $M$ by $M$ identity matrix. For notational simplicity, we drop the subscripts of the matrices when there is no confusion.

\section{B. Relaying Strategy}

At the $i$-th relay, $\boldsymbol{y}_{i}=\left[\boldsymbol{y}_{i, 1}^{T}, \boldsymbol{y}_{i, g}^{T}, \boldsymbol{y}_{i, 2}^{T}\right]^{T}=h_{i} \overline{\boldsymbol{x}}+\boldsymbol{n}_{i}$ is received in which $\boldsymbol{n}_{i}$ are independent complex white Gaussian random vectors with zero mean and autocorrelation matrices $\sigma_{i}^{2} \boldsymbol{I}_{\left(2 M+M_{p}\right)}, \boldsymbol{y}_{i, j}=h_{i} \boldsymbol{x}_{j}+\boldsymbol{n}_{i, j}, \boldsymbol{y}_{i, g}=\boldsymbol{n}_{i, g}$ and $h_{i}$ are independent circularly symmetric complex Gaussian random variables with zero mean and variance 0.5 per dimension. Then, based on the relaying strategy given in Table I, $\boldsymbol{s}_{i}=\left[\boldsymbol{s}_{i, 1}^{T}, \boldsymbol{s}_{i, g}^{T}, s_{i, 2}^{T}\right]^{T}$ of length $2 M+M_{p}$ is forwarded to the destination in which $\zeta($.$) denotes the time reversal operation,$ i.e., $\zeta\left(\left[z_{0}, \ldots, z_{N-1}\right]\right)=\left[z_{N-1}, \ldots, z_{0}\right],(.)^{*}$ denotes the complex conjugation operation and $z[a: b]=\left[z_{a}, \ldots, z_{b}\right] . \boldsymbol{s}_{i, 1}$ and $\boldsymbol{s}_{i, 2}$ are of length $M$ and $\boldsymbol{s}_{1, g}$ is of length $M_{p}$. Note that, the infix block forwarded by the relay nodes corresponds to the noisy $\mathrm{CP}$ of the second block and noisy cyclic suffix of the first block. We can also represent the forwarded signals from the relay nodes by $\boldsymbol{s}_{1}=\boldsymbol{R}_{1} \boldsymbol{y}_{1}$ and $\boldsymbol{s}_{2}=\boldsymbol{R}_{2} \boldsymbol{y}_{2}^{*}$, where

$$
\begin{aligned}
& \boldsymbol{R}_{1}=\left[\begin{array}{c:c:cccc}
\boldsymbol{I}_{M} & \mathbf{0}_{M \times M_{p}} & \mathbf{0} \\
\hdashline \boldsymbol{I}_{M_{p}} \mathbf{0} & \mathbf{0}_{M_{p} \times M_{p}} & \mathbf{0} & \boldsymbol{I}_{M_{p}} \\
\hdashline \mathbf{0} & \mathbf{0}_{M \times M_{p}} & \boldsymbol{I}_{M}
\end{array}\right], \\
& \boldsymbol{R}_{2}=\left[\begin{array}{ccc|cc|cc}
- & \mathbf{0} & & \mathbf{0}_{M \times M_{p}} & \multicolumn{1}{c}{\boldsymbol{J}_{M}} \\
-\boldsymbol{J}_{M_{p}} & \mathbf{0} & - & -\mathbf{0}_{M_{p} \times M_{p}} & \mathbf{0} & \boldsymbol{J}_{M_{p}} \\
-\overline{\boldsymbol{J}}_{M} & - & - & - & \mathbf{0}_{M \times M_{p}} & & \mathbf{0}
\end{array}\right],
\end{aligned}
$$

with $\boldsymbol{J}_{M}$ denoting an $M$ by $M$ anti-diagonal identity matrix.

\section{Signal Detection Techniques}

In this section, we first provide the signaling structure at the destination of the considered system for general case $(|d| \leq 0.5)$, then focus on the case of $d=0$ and present the optimal ML and sub-optimal detectors. Furthermore, we provide a discussion on acceptable values of $d$ to obtain reasonable performance.

\section{A. Received Signal at the Destination}

Without loss of generality, we focus on $D+d \geq 0$. At the destination node, for the received signal, we have

$$
\begin{aligned}
\boldsymbol{y} & =g_{1} \boldsymbol{s}_{1}+\alpha g_{2} \boldsymbol{s}_{2}^{D}+\boldsymbol{w}+\boldsymbol{n}_{3} \\
& =g_{1} \boldsymbol{R}_{1}\left(h_{1} \overline{\boldsymbol{x}}+\boldsymbol{n}_{1}\right)+\alpha g_{2}\left(\boldsymbol{R}_{2}\left(h_{2}^{*} \overline{\boldsymbol{x}}^{*}+\boldsymbol{n}_{2}^{*}\right)\right)^{D}+\boldsymbol{w}+\boldsymbol{n}_{3} \\
& =g_{1} h_{1} \boldsymbol{R}_{1} \boldsymbol{Q} \boldsymbol{x}+\alpha g_{2} h_{2}^{*}\left(\boldsymbol{R}_{2} \boldsymbol{Q}\right)^{D} \boldsymbol{x}^{*}+\boldsymbol{w}+\boldsymbol{n},
\end{aligned}
$$

in which $\boldsymbol{s}_{2}^{D}=\left[\mathbf{0}_{1 \times D}, s_{2,0}, \cdots, s_{2,2 M+M_{p}-D-1}\right]^{T}, \quad \boldsymbol{n}_{3}$ is a white Gaussian random vector with zero mean and autocorrelation matrix $\sigma_{3}^{2} \boldsymbol{I}_{\left(2 M+M_{p}\right)}, \alpha=\frac{\operatorname{sinc}(d) \cos \beta \pi d}{1-4 \beta^{2} d^{2}}$, $\boldsymbol{n}=g_{1} \boldsymbol{R}_{1} \boldsymbol{n}_{1}+g_{2} \boldsymbol{R}_{2}^{D} \boldsymbol{n}_{2}^{*}+\boldsymbol{n}_{3}$,

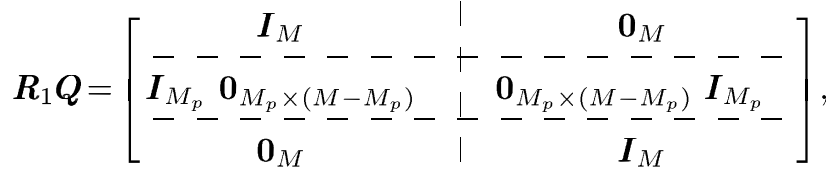

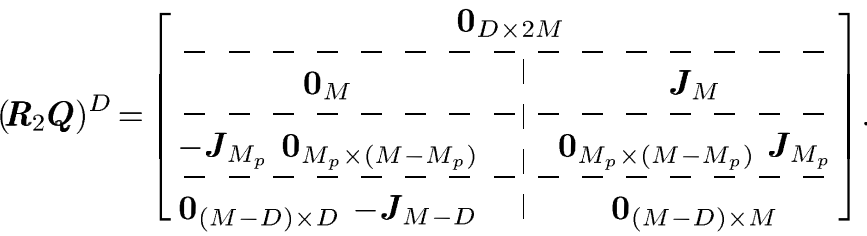

Furthermore, $\boldsymbol{w}$ represents the ISI experienced for non-zero values of $d$. Let us only consider the ISI effect of the first adjacent symbol, then we have $\boldsymbol{w}=\gamma g_{2} h_{2}^{*}\left(\boldsymbol{R}_{2} \boldsymbol{Q}\right)^{D+\operatorname{sign}(d)} \boldsymbol{x}^{*}$ for where $\operatorname{sign}(d)$ is the signum function and $\gamma=$ $\operatorname{sinc}(1-|d|) \frac{\cos \beta \pi(1-|d|)}{1-4 \beta^{2}(1-|d|)^{2}}$. The first $D$ samples in $\boldsymbol{y}$ are corrupted by interblock interference (IBI) and $y_{j}$ for $j=\left\{M+D, \ldots, M+M_{p}-1\right\}$ are the overhead symbols, therefore we truncate the first $D$ samples and $y_{M+D}$ to $y_{M+M_{p}-1}$, and define a new vector as $\boldsymbol{y}^{\prime}=\left[\boldsymbol{y}_{1}^{\boldsymbol{\prime}_{1}^{T}}, \boldsymbol{y}_{2}^{\boldsymbol{\prime}^{T}}\right]^{T}$ with $\boldsymbol{y}_{1}^{\prime}=\left[y_{D}, \ldots, y_{M-1}, y_{M}^{*}, \ldots, y_{M+D-1}^{*}\right]^{T}$ and $\boldsymbol{y}_{2}{ }_{2}=$ $\left[y_{M+M_{p}}, \ldots, y_{M+M_{p}+D-1}, y_{M+M_{p}+D}^{*}, \ldots, y_{2 M+M_{p}-1}^{*}\right]^{T}$. Note that after truncation some information may be lost which can be extracted out by employing a more complex joint detector, however, joint detection over more than two successive blocks is out of the focus of this work. To simplify the derivations, we assume $M_{p}=D$ from now on with understanding that for $M_{p}>D$, after truncating $y_{j}$ for $j=\left\{M+D, \ldots, M+M_{p}-1\right\}$, the performance analysis 
would be the same. Furthermore, by defining $C_{1}=g_{1} h_{1}$, $C_{2}=\alpha g_{2} h_{2}^{*}, \boldsymbol{x}_{i}^{\prime}=\left[x_{i, 0}^{*}, \ldots, x_{i, D-1}^{*}, x_{i, D}, \ldots, x_{i, M-1}\right]^{T}$ and

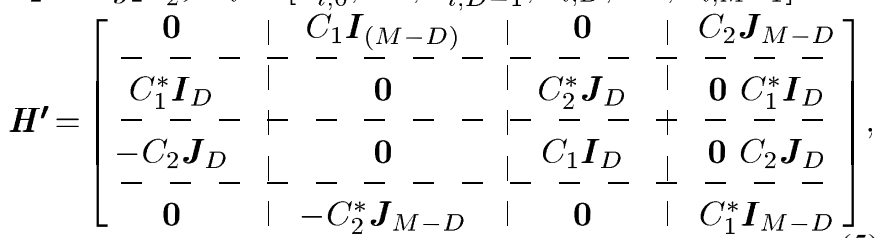
we can write $\boldsymbol{y}^{\prime}=\boldsymbol{H}^{\prime}\left[\begin{array}{c}\boldsymbol{x}^{\prime}{ }_{1} \\ \left(\boldsymbol{x}_{2}\right)^{*}\end{array}\right]+\boldsymbol{w}^{\prime}+\boldsymbol{n}^{\prime}$, where $\boldsymbol{n}^{\prime}$ is the colored Gaussian noise with the autocorrelation matrix $\boldsymbol{K}_{n}$ given as the first equation shown at the bottom of the page, with $\sigma^{2}=$ $\left|g_{1}\right|^{2} \sigma_{1}^{2}+\left|g_{2}\right|^{2} \sigma_{2}^{2}+\sigma_{3}^{2}$.

\section{B. Maximum Likelihood Detector}

Let us first focus on $d=0$, i.e., ISI free case with $\alpha=1$ and $\beta=0$. Since $\boldsymbol{n}^{\prime}$ is colored Gaussian noise, in order to obtain the ML detector, we first need to whiten the noise. After noise whitening (see [9] for details), we arrive at

$$
\hat{\boldsymbol{x}}^{\prime}=\underset{\boldsymbol{x}^{\prime}}{\arg \max } \operatorname{Re}\left\{\left(\boldsymbol{H} \boldsymbol{y}^{\prime}\right)^{H} \boldsymbol{x}^{\prime}\right\}-\frac{\sigma^{2}}{2} \eta_{x}^{\prime},
$$

with $\eta_{x}^{\prime}=\boldsymbol{x}^{\prime{ }^{H}} \boldsymbol{H}^{\prime}{ }^{H} \boldsymbol{K}_{n}^{-1} \boldsymbol{H}^{\prime} \boldsymbol{x}^{\prime}$ and $\boldsymbol{H}=\sigma^{2} \boldsymbol{H}^{{ }^{H}} \boldsymbol{K}_{n}^{-1}$ as given in (7), at the bottom of the page, in which $\alpha_{i}^{2}=\frac{\sigma^{2}\left|g_{i}\right|^{2} \sigma_{i}^{2}}{\sigma^{4}+\sigma^{2} \sigma_{i}^{2}\left|g_{i}\right|^{2}-\sigma_{i}^{4}\left|g_{i}\right|^{4}}$ and $\beta_{i}^{2}=\frac{\sigma^{4}}{\sigma^{4}+\sigma^{2} \sigma_{i}^{2}\left|g_{i}\right|^{2}-\sigma_{i}^{4}\left|g_{i}\right|^{4}}$. Defining $\boldsymbol{y}_{k}=\left[y_{2 D-k-1}, y_{M+k}^{*}, y_{M+2 D-k-1}, y_{2 M+k}^{*}\right]^{T}$ and $\boldsymbol{x}_{k}=\left[x_{1, k}^{*}, x_{1,2 D-k-1}, x_{2, D-k-1}, x_{2, M-D+k}^{*}\right]^{T}$ leads to $\hat{\boldsymbol{x}}^{\prime}=\underset{\boldsymbol{x}^{\prime}}{\arg \max } \operatorname{Re}\left\{\sum_{j=\mathbf{0}}^{M-2 D-1}\left(\boldsymbol{H}_{1}\left[\begin{array}{c}y_{2 D+j} \\ y_{2 M-j-1}\end{array}\right]\right)^{H}\left[\begin{array}{c}x_{1, j+2 D} \\ x_{2, M-j-D-1}^{*}\end{array}\right]\right.$ $\left.+\sum_{k=\mathbf{0}}^{D-1}\left(\boldsymbol{H}_{2} \boldsymbol{y}_{k}\right)^{H} \boldsymbol{x}_{k}\right\}-\frac{\sigma^{2}}{2} \eta_{x}^{\prime}$,

where $\boldsymbol{H}_{1}=\left[\begin{array}{cc}C_{1}^{*} & -C_{2} \\ C_{2}^{*} & C_{1}\end{array}\right]$ and $\boldsymbol{H}_{2}$ is given as the last equation at the bottom of the page.

The above ML detection criterion can be split into several independent detection criteria. More precisely $\left[x_{1, j+2 D}, x_{2, M-j-D-1}\right]^{T}$ for $j \in\{0, \ldots, M-2 D-1\}$ and $\boldsymbol{x}_{k}$ for $k \in\{0, \cdots, D-1\}$, can be detected separately. For $j \in\{0, \ldots, M-2 D-1\}$, the ML detector yields

$$
\begin{aligned}
{\left[\begin{array}{c}
\hat{x}_{1, j+2 D} \\
\hat{x}_{2, M-j-D-1}
\end{array}\right]=} & \underset{\left[x_{1}, x_{2}\right]^{T}}{\arg \max } \operatorname{Re}\left\{\left(\boldsymbol{H}_{1}\left[\begin{array}{c}
y_{2 D+j} \\
y_{2 M-j-1}
\end{array}\right]\right)^{H}\left[\begin{array}{l}
x_{1} \\
x_{2}^{*}
\end{array}\right]\right\} \\
& -\frac{\sigma^{2}}{2}\left(\left|C_{1}\right|^{2}+\left|C_{2}\right|^{2}\right)\left(\left|x_{1}\right|^{2}+\left|x_{2}\right|^{2}\right)
\end{aligned}
$$

which represents an Alamouti detection structure and it clearly shows that the system achieves full transmit diversity in detecting $\left[x_{1, j+2 D}, x_{2, M-j-D-1}\right]^{T}(j \in\{0, \ldots, M-2 D-1\})$ independent of the delay value $D$. Furthermore, for $k \in\{0, \ldots, D-1\}$, the optimal detector is obtained as

$$
\hat{\boldsymbol{x}}_{k}=\underset{\boldsymbol{x}_{k}}{\arg \max } \operatorname{Re}\left\{\left(\boldsymbol{H}_{2} \boldsymbol{y}_{k}\right)^{H} \boldsymbol{x}_{k}\right\}-\frac{\sigma^{2}}{2} \boldsymbol{x}_{k}^{H} \boldsymbol{H}_{3} \boldsymbol{x}_{k},
$$

where $\boldsymbol{H}_{3}$ is given in (11) at the bottom of the next page. Through numerical examples, in Section $\mathrm{V}$, we show that the optimal detector achieves full diversity in detection of all the symbols.

\section{A Sub-Optimal Detector}

As explained in Section IV-B, to obtain the optimal detector we first need to whiten the noise which results in (9) and (10). In this section, we propose a sub-optimal detector with a smaller computational complexity in comparison with the optimal detector. By considering only the first and last $M-D$ samples of $\boldsymbol{y}^{\prime}$, we obtain $(j \in\{0, \ldots, M-D-1\})$

$$
\left[\begin{array}{c}
y_{D+j} \\
y_{2 M+D-j-1}^{*}
\end{array}\right]=\boldsymbol{H}_{1}^{H}\left[\begin{array}{c}
x_{1, j+D} \\
x_{2, M-j-1}^{*}
\end{array}\right]+\left[\begin{array}{c}
n_{D+j} \\
n_{2 M+D-j-1}
\end{array}\right],
$$

where $n_{D+j}$ and $n_{2 M+D-j-1}$ are i.i.d. complex Gaussian noise with zero mean and variance $\sigma^{2}$. The above expression shows the Alamouti structure for $\left[x_{1, j+D}, x_{2, M-j-1}\right]^{T}$ $(j \in\{0, \ldots, M-D-1\})$, therefore we can employ fast ML detection to obtain the estimate $\left[\hat{x}_{1, j+D}, \hat{x}_{2, M-j-1}\right]^{T}$ for $j \in\{0, \ldots, M-D-1\}$. Note that by employing the optimal detector we arrive at the fast ML detection for $\left[x_{1, j+D}, x_{2, M-j-1}\right]^{T}(j \in\{0, \ldots, M-2 D-1\})$. Furthermore, for the remaining $2 D$ symbols, we have $(k \in\{0, \ldots, D-1\})$

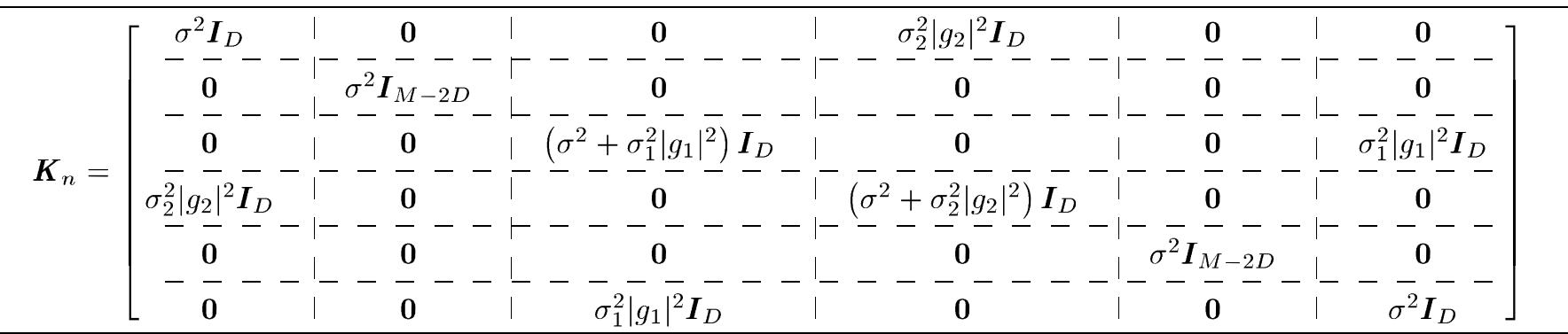

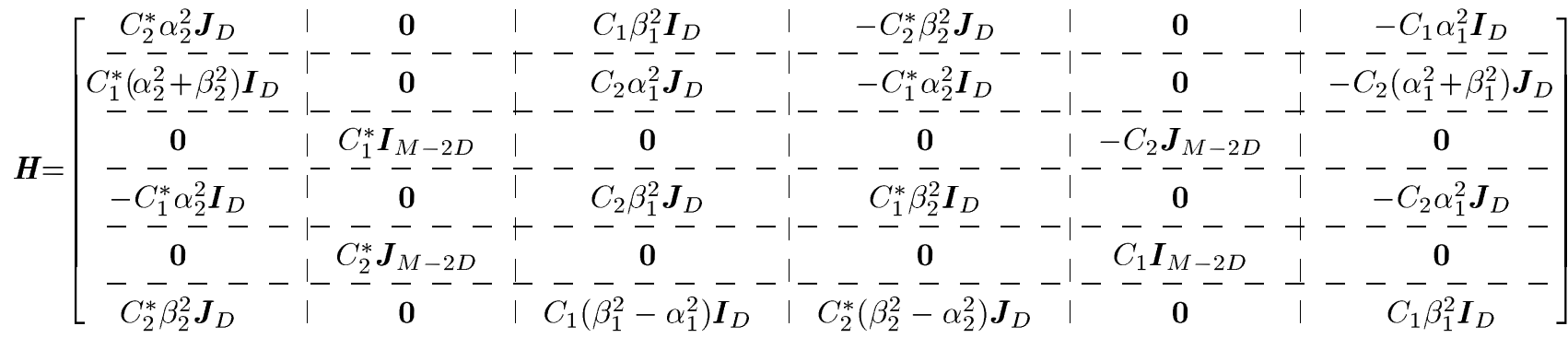

$$
\begin{aligned}
& \boldsymbol{H}_{2}=\left[\begin{array}{cccc}
C_{2}^{*} \alpha_{2}^{2} & C_{1} \beta_{1}^{2} & -C_{2}^{*} \beta_{2}^{2} & -C_{1} \alpha_{1}^{2} \\
C_{1}^{*}\left(\alpha_{2}^{2}+\beta_{2}^{2}\right) & C_{2} \alpha_{1}^{2} & -C_{1}^{*} \alpha_{2}^{2} & -C_{2}\left(\alpha_{1}^{2}+\beta_{1}^{2}\right) \\
-C_{1}^{*} \alpha_{2}^{2} & C_{2} \beta_{1}^{2} & C_{1}^{*} \beta_{2}^{2} & -C_{2} \alpha_{1}^{2} \\
C_{2}^{*} \beta_{2}^{2} & C_{1}\left(\beta_{1}^{2}-\alpha_{1}^{2}\right) & C_{2}^{*}\left(\beta_{2}^{2}-\alpha_{2}^{2}\right) & C_{1} \beta_{1}^{2}
\end{array}\right]
\end{aligned}
$$


the second equation shown at the bottom of the page, where $n_{M+k}$ and $n_{M+2 D-k-1}$ are independent complex Gaussian noise with zero means and variances of $\sigma^{2}+\sigma_{1}^{2}\left|g_{1}\right|^{2}$ and $\sigma^{2}+\sigma_{2}^{2}\left|g_{2}\right|^{2}$, respectively. Employing the detected symbol $\hat{x}_{2, M-D+k}$ to mitigate the ISI effect of $x_{2, M-D+k}$, we obtain

$$
\begin{aligned}
& {\left[\begin{array}{c}
y_{M+k}^{*} \\
y_{M+2 D-k-1}
\end{array}\right]-\left[\begin{array}{l}
C_{1}^{*} \\
C_{2}
\end{array}\right] \hat{x}_{2, M-D+k}^{*}=\boldsymbol{H}_{1}^{T}\left[\begin{array}{c}
x_{1, k}^{*} \\
x_{2, D-k-1}
\end{array}\right]} \\
& +\left[\begin{array}{c}
C_{1}^{*} \\
C_{2}
\end{array}\right]\left(x_{2, M-D+k}-\hat{x}_{2, M-D+k}\right)^{*}+\left[\begin{array}{c}
n_{M+k}^{*} \\
n_{M+2 D-k-1}
\end{array}\right],
\end{aligned}
$$

which exhibits an Alamouti coding structure. Obviously, if $x_{2, M-D+k}$ is detected correctly, the ISI is perfectly removed, otherwise the signal is even more corrupted. Therefore, we expect that for higher SNR values, the sub-optimal detector performance becomes closer to that of the optimal detector.

\section{Comments on Non-Integer Delay Values $(d \neq 0)$}

When $d \neq 0$, ISI is introduced into the system which degrades the performance. Defining $\boldsymbol{w}^{\prime}=\left[w_{0}, \ldots, w_{2 M-1}\right]$, we have $\left|w_{j}\right|^{2}=\gamma^{2}\left|g_{2}\right|^{2}\left|h_{2}\right|^{2}$ for $j \in\{0, \cdots, M+\operatorname{sign}(d)-$ $1\}$ and $j \in\{M+D+\operatorname{sign}(d), \cdots, 2 M-1\}$ and $\left|w_{j}\right|^{2} \leq$ $2 \gamma^{2}\left|g_{2}\right|^{2}\left|h_{2}\right|^{2}$ for $j \in\{M+\operatorname{sign}(d), \cdots, M+D+\operatorname{sign}(d)-1\}$. Therefore, if we approximate the ISI as complex Gaussian noise with zero mean and variance of $\left|w_{j}\right|^{2}$, the instantaneous SNR can be approximated as $S N R_{\text {inst }}=\frac{2\left(\left|g_{1}\right|^{2}\left|h_{1}\right|^{2}+\left.\alpha^{2} g_{2}\right|^{2}\left|h_{2}\right|^{2}\right)}{N_{0}\left(\left|g_{1}\right|^{2}+\left|g_{2}\right|^{2}+1\right)+\left|w_{j}\right|^{2}}$. Let us consider the low noise power case where $S N R_{\text {inst }} \approx$ $\frac{\alpha^{2}}{\gamma^{2}}+\frac{\left.\left|g_{1}{ }^{2}\right| h_{1}\right|^{2}}{\gamma^{2}\left|g_{2}\right|^{2}\left|h_{2}\right|^{2}}$. We observe that to obtain reasonable performance, we need to have $\alpha^{2} \gg \gamma^{2}$. For example, for $\beta=0.5$, to have $\frac{\alpha^{2}}{\gamma^{2}} \geq 100$, we need $|d| \leq 0.1$.

\section{E. Comments on Implementation and Detection Complexity}

Let us assume that the source node transmits waveforms of length $T$ seconds. At the first relay, to generate the affix block, the first $M_{p} T$ seconds and the last $M_{p} T$ seconds of the received signal need to be added together which can be implemented in the analog domain. To do so, $R_{1}$ suffers a latency of $M T$ seconds. At the second relay, adding the sign changed version of the first $M_{p} T$ seconds of the received signal to the last $M_{p} T$ seconds of the received signal generates the affix block, however, we still need to perform time reversal and complex conjugation. Time reversal introduces a latency of $2 M T$ seconds for which we first need to store the received signal corresponding to the two consecutive blocks along with the generated affix block, then forward the time reversed version of the signal. Complex conjugation may also be implemented in analog domain by first separating the in-phase and quadrature components of the signal then combining them by changing the sign of the quadrature component.

To compare the computational complexity of the proposed optimal and sub-optimal detectors, we count the number of real multiplications required in detection of QPSK modulated symbols. We assume that the common variables in detection of every two blocks are computed only once and buffered at the receiver. Therefore, in employing the optimal detector $72 M+12688 D+$ 20 real multiplications are required while in employing the sub-

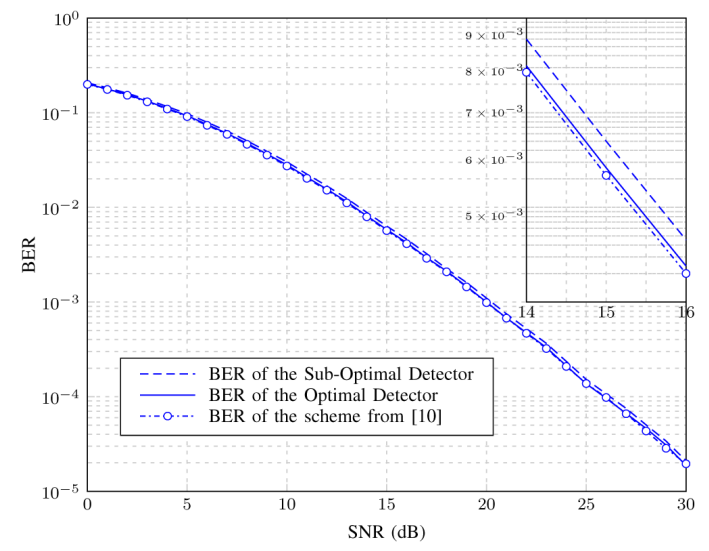

Fig. 2. BER performance of the proposed scheme for both optimal and suboptimal detectors and the scheme from [10].

optimal detector we only need to perform $72 M+4 D+4$ real multiplications.

\section{Simulation Results}

In this section, we provide numerical examples to study the ability of the proposed scheme in combating the asynchronism issues in cooperative communication systems. We consider BPSK modulated transmission of the data blocks of length $M=128$ with an affix of length $M_{p}=64$ where $d=0$ (no ISI) and $D$ is uniformly distributed over $\{0, \ldots, 64\}$ and $h_{i}$ and $g_{i}$ are i.i.d. complex Gaussian random variables with zero mean and unit variance. We also assume that $\sigma_{1}^{2}=\sigma_{2}^{2}=\sigma_{3}^{2}=\frac{N_{0}}{2}$ and define the average signal to noise ratio at the receiver as $E\left\{\frac{2\left(\left|g_{1}\right|^{2}\left|h_{1}\right|^{2}+\left|g_{2}\right|^{2} \mid h_{2}{ }^{2}\right)}{N_{0}\left(\left.{ }^{\prime} g_{1}\right|^{2}+\left|g_{2}\right|^{2}+1\right)}\right\} \approx \frac{1.192694}{N_{0}}$

In Fig. 2, we compare the BER performance of the proposed scheme for both optimal and sub-optimal detectors with the BER performance of the scheme proposed in [10] in which all the received symbols see a perfect Alamouti structure as in (9). We observe that the difference between the performance of the optimal detector and the scheme from [10] is less than $0.05 \mathrm{~dB}$. Furthermore, the sub-optimal detector performs very close to the optimal detector and the scheme from [10]. The proposed transmission scheme improves the rate by $20 \%$ at the cost of a performance loss by about $0.2 \mathrm{~dB}$ with respect to the one in [10].

\section{CONCLUSIONS}

We proposed a new TR-STBC scheme useful in achieving asynchronous cooperative diversity under flat fading channel conditions which achieves a higher spectral efficiency than the existing STBC schemes in the literature. More precisely, to combat the asynchronism issues arising from relative delays among the signals received from different relays, a smaller overhead needs to be added in transmitting every pair of data blocks compared with the existing schemes. We obtained the optimal detector structure and proposed a sub-optimal detector with a smaller computational complexity. Numerical examples demonstrate that the proposed scheme achieves full diversity for both optimal and sub-optimal detectors.

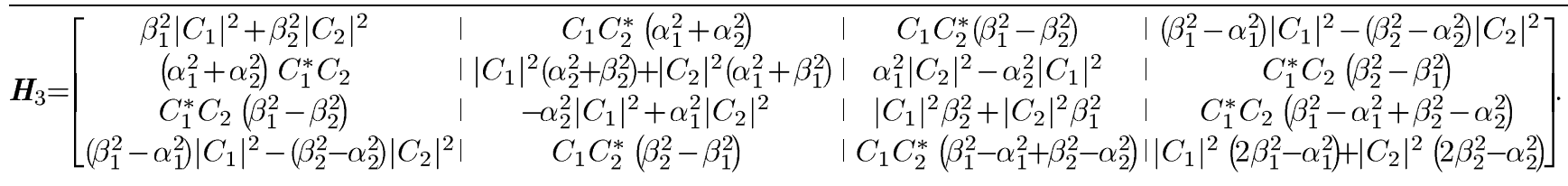

$$
\left[\begin{array}{c}
y_{M+k}^{*} \\
y_{M+2 D-k-1}
\end{array}\right]=\boldsymbol{H}_{1}^{T}\left[\begin{array}{c}
x_{1, k}^{*} \\
x_{2, D-k-1}
\end{array}\right]+\left[\begin{array}{c}
C_{1}^{*} \\
C_{2}
\end{array}\right] x_{2, M-D+k}^{*}+\left[\begin{array}{c}
n_{M+k}^{*} \\
n_{M+2 D-k-1}
\end{array}\right]
$$




\section{REFERENCES}

[1] S. Alamouti, "A simple transmit diversity technique for wireless communications," IEEE J. Sel. Areas Commun., vol. 16, no. 8, pp. 1451-1458, Oct. 1998.

[2] J. Laneman and G. Wornell, "Distributed space-time-coded protocols for exploiting cooperative diversity in wireless networks," IEEE Trans. Inf. Theory, vol. 49, no. 10, pp. 2415-2425, Oct. 2003.

[3] X. Li, "Space-time coded multi-transmission among distributed transmitters without perfect synchronization," IEEE Signal Process. Lett., vol. 11, no. 12, pp. 948-951, Dec. 2004.

[4] Y. Li and X. Xia, "Full diversity distributed space-time trellis codes for asynchronous cooperative communications," in Proc. IEEE Int. Symp. Inf. Theory (ISIT), 2005, pp. 911-915.

[5] Z. Zhong, S. Zhu, and A. Nallanathan, "Delay tolerant distributed linear convolutional space-time code with minimum memory length under frequency-selective channels," IEEE Trans. Wireless Commun., vol. 8, no. 8, pp. 3944-3949, Aug. 2009.
[6] Z. Li, X.-G. Xia, and M. H. Lee, "A simple orthogonal space-time coding scheme for asynchronous cooperative systems for frequency selective fading channels," IEEE Trans. Commun., vol. 58, no. 8, pp. 2219-2224, Aug. 2010.

[7] M. Vajapeyam, S. Vedantam, U. Mitra, J. C. Preisig, and M. Stojanovic, "Distributed space-time cooperative schemes for underwater acoustic communications," IEEE J. Oceanic Eng., vol. 33, no. 4, pp. 489-501, Oct. 2008 .

[8] X. Li, C. Xing, Y.-C. Wu, and S. C. Chan, "Timing estimation and resynchronization for amplify-and-forward communication systems," IEEE Trans. Signal Process., vol. 58, no. 4, pp. 2218-2229, Apr. 2010.

[9] M. Rahmati, "On asynchronous communication systems: Capacity bounds and relaying schemes," Ph.D. dissertation, Arizona State Univ., Tempe, May 2013 [Online]. Available: http://hdl.handle.net/ 2286/R.I.17865

[10] Z. Li and X.-G. Xia, "A simple Alamouti space-time transmission scheme for asynchronous cooperative systems," IEEE Signal Process. Lett., vol. 14, no. 11, pp. 804-807, Nov. 2007. 